\title{
Composite SAR Imaging Using Sequential Joint Sparsity ${ }^{\text {th }}$
}

\author{
Toby Sanders ${ }^{1}$, Anne Gelb ${ }^{2}$, Rodrigo B. Platte ${ }^{1}$
}

\begin{abstract}
This paper investigates accurate and efficient $\ell_{1}$ regularization methods for generating synthetic aperture radar (SAR) images. Although $\ell_{1}$ regularization algorithms are already employed in SAR imaging, practical and efficient implementation in terms of real time imaging remain a challenge. Here we demonstrate that fast numerical operators can be used to robustly implement $\ell_{1}$ regularization methods that are as or more efficient than traditional approaches such as back projection, while providing superior image quality. In particular, we develop a sequential joint sparsity model for composite SAR imaging which naturally combines the joint sparsity methodology with composite SAR. Our technique, which can be implemented using standard, fractional, or higher order total variation regularization, is able to reduce the effects of speckle and other noisy artifacts with little additional computational cost. Finally we show that generalizing total variation regularization to non-integer and higher orders provides improved flexibility and robustness for SAR imaging.

Keywords: Synthetic Aperture Radar, Image Reconstruction, $\ell_{1}$ Regularization, Joint Sparsity 2010 MSC: 00-01, 99-00
\end{abstract}

\section{Introduction}

Synthetic aperture radar (SAR) is an all weather, night and day imaging technique where image reconstruction is computed from electromagnetic scattering data. The reconstructed image represents a 2-D or 3-D visual of the scattering objects within the scene. While technological and computational advances have continually improved the quality of SAR images, there are still

\footnotetext{
This work is supported in part by the grants NSF-DMS 1502640 and AFOSR FA9550-15-1-0152.

Email addresses: toby.sanders@asu.edu (Toby Sanders), Anne.E.Gelb@Dartmouth.edu (Anne Gelb), rbpœasu.edu (Rodrigo B. Platte)

${ }^{1}$ School Of Mathematical and Statistical Sciences, Arizona State University, P.O. Box 871804, Tempe, AZ, 852871804, USA

${ }^{2}$ Department of Mathematics, Dartmouth College, Hanover, NH, 03755, USA
}

Preprint submitted to Journal of LATEX Templates

February 27, 2017

(C) 2017. This manuscript version is made available under the Elsevier user license http://www.elsevier.com/open-access/userlicense/1.0/ 
lingering challenges. Among those are issues of specular noise, sidelobe artifacts, and auto-focusing of data. Moreover, modern computational algorithms used for reconstructing SAR images also face several distinct challenges, namely the large data sets that are acquired at suboptimal sampling patterns must be processed in a timely and effective manner. In addition, as a result of the digitization of an underlying continuous scene, speckle noise is inherent in standard SAR imaging techniques such as back projection. In recent years, $\ell_{1}$ regularization methods, also known as compressive sensing, have been used to effectively reduce these noise artifacts [10, 7, 29]. However, run time and practical implementation are not always considered. Finally, for wide-angular SAR, there is also a concern with angular dependence of the scattered response. In particular, the energy of the incoming incident wave is scattered off objects in the scene front and thus not evenly mixed with the full imaging scene. To overcome this issue, a method known as composite image formation, where small narrow angular patches of the data are considered separately to eventually form a final ideal image, has been successfully introduced in [25, 11, 28]. We note that $\ell_{1}$ regularization was used as an additional post-processing procedure in [25, 11.

This investigation discusses many of the practical concerns involved with $\ell_{1}$ regularization that are particular to $\mathrm{SAR}^{3}$. Specifically, the nature and amount of data dictate special consideration to ensure efficiency and accuracy. We are able to implement an $\ell_{1}$ regularization algorithm that yields at least as good as and often better run times as traditional back projection. Moreover, we demonstrate that our design enables better handling of noise and other unwanted artifacts often found in currently used algorithms. With regard to composite imaging, we are further able to reduce the effects of undesirable artifacts by designing an improved sequential joint sparsity technique that exploits the overlapping support of the recovered sequence of images. As in the case of wide-angular SAR, we demonstrate both efficiency and accuracy of our approach.

Our regularization can be implemented using standard or high order total variation, [13. Our transformation in the regularization term is defined in [6] as an edge detector with (higher) integer order of accuracy away from a discontinuities, and used in [5, 34] for image reconstruction from Fourier and tomographic data. We demonstrate that generalizing the sequential joint sparsity methodology to continuous orders (including fractional [16]) enables greater flexibility and

\footnotetext{
${ }^{3}$ We limit this investigation to spotlight SAR, although we anticipate that our algorithms may also be used for other SAR modalities.
} 
robustness for SAR imaging.

35

The rest of this paper is organized as follows. In Section 2 we describe the continuous far field imaging model. In Section 3 we discuss the discretization of the model and the current state of the art tools needed to solve the inverse problem, namely the non-uniform fast Fourier transform (NUFFT). We then present the general regularized inverse model, and adapt it to fit the particular concerns associated with SAR. We specifically focus on phase estimation, parameter selection, and convergence. Section 4 considers composite imaging, which leads us to the main contribution of this investigation, our joint sparsity model derived in Section 5. We also generalize our approach to consider different $\ell_{1}$ sparsity transforms, in particular higher and fractional order total variation. Numerical experiments are provided throughout to demonstrate the accuracy and efficiency of our new algorithm. A summary of our findings is provided in Section 6 .

\section{2. Far Field SAR Imaging Problem}

SAR data are acquired by measuring reflected waveforms along some known flight path. In spotlight SAR, the waveforms are high bandwidth pulses transmitted from the flight locations, at azimuth angles, $\theta$, typically measured at equal increments. The ground scene is assumed to be a circular region of radius $R$ given by $\mathcal{D}=\left\{(x, y) \mid x^{2}+y^{2} \leq R^{2}\right\}$. For each angle $\theta$, the emitted pulses are linear FM chirp signals given by the real part of

$$
s(t)= \begin{cases}e^{i\left(\omega_{0} t+\alpha t^{2}\right)}, & |t| \leq \frac{T}{2} \\ 0, & \text { otherwise }\end{cases}
$$

where $\omega_{0}$ is the center or carrier frequency, $2 \alpha$ is the chirp rate, and $T>0$ is the chirp pulse duration. The chirp signal is essentially mixed or convolved with the reflectivity function, $f(x, y)$, and the reflected waveforms are recorded at a equally spaced discrete time steps. Our investigation relies on the following key assumptions:

1. The chirp rate $\alpha$ is small enough so that for each recorded time $t$, the reflected waveform after demodulation is effectively mixed with the wave having instantaneous frequency $\omega(t)=$ $\omega_{0}+2 \alpha\left(t-2 d_{\theta} / c\right),[22]$. Here $d_{\theta}$ is the distance from the flight location to the scene center at angle $\theta$, and $c$ is the speed of light. 
2. The distance of the flight path to the receiver is assumed to be sufficiently larger than $R$, so that the range error due to wavefront curvature is negligible.

3. The scene contains only isotropic scatterers, which implies that the response amplitude $f(x, y)$ is independent of the viewing angle $\theta$.

4. There are no scatterers outside $\mathcal{D}$.

We note that assumptions 1, 2, and 4 are commonly used in far field SAR as they introduce little observable error. While assumption 3 is generally not true, it is reasonable for modeling narrowangle SAR. We discuss other techniques for handling this issue for the wide-angle case in Section 4.

Finally then, given some constant angle of elevation $\phi$ between the flight path and $\mathcal{D}$, the reflected waveforms take the form

$$
\hat{f}(\omega(t), \theta)=\iint_{\mathcal{D}} f(x, y) \exp \left\{-i \frac{4 \pi \omega(t) \cos \phi}{c}(x, y) \cdot(\cos \theta, \sin \theta)\right\} d x d y,
$$

where again $c$ is the speed of light. For each fixed $\theta$, this return is simply the Fourier transform of $f$ through a single slice at angle $\theta$, where the frequency values are away from the origin.

If we let $p_{\theta} f$ denote the projection of $f$ at angle $\theta$, which may be defined by

$$
\left(p_{\theta} f\right)(x)=\int_{-R}^{R} f\left((x, y) Q_{\theta}^{T}\right) d y, \quad \text { where } \quad Q_{\theta}=\left[\begin{array}{rr}
\cos \theta & -\sin \theta \\
\sin \theta & \cos \theta
\end{array}\right]
$$

then by the Fourier slice theorem we have

$$
\hat{f}(\omega(t), \theta)=\int_{-R}^{R}\left(p_{\theta} f\right)(x) \exp \left\{-i \frac{4 \pi \omega(t) \cos \phi}{c} x\right\} d x .
$$

\section{Inverse Methods for SAR}

\subsection{Discretization}

To discretize the problem, we consider a set of azimuth angles, $\left\{\theta_{j}\right\}_{j=1}^{N}$, and a set of time steps corresponding to a discrete set of frequency values, $\left\{\omega_{k}\right\}_{k=1}^{K}$. Let $\hat{\mathbf{f}}_{\theta_{j}}$ be the vector containing the $K$ frequency samples at angle $\theta_{j}$ and $\mathbf{f} \in C^{n^{2}}$ be our vectorized $n \times n$ complex scene to be recovered. 
Stacking the angular data into a vector, the discretized SAR equation becomes

$$
\hat{\mathbf{f}}=F A \mathbf{f},
$$

where

$$
\hat{\mathbf{f}}=\left[\begin{array}{c}
\hat{\mathbf{f}}_{\theta_{1}} \\
\hat{\mathbf{f}}_{\theta_{\mathbf{2}}} \\
\vdots \\
\hat{\mathbf{f}}_{\theta_{\mathbf{N}}}
\end{array}\right], \quad A=\left[\begin{array}{c}
P_{\theta_{1}} \\
P_{\theta_{2}} \\
\vdots \\
P_{\theta_{N}}
\end{array}\right],
$$

and $F$ is a block diagonal matrix where each block performs the 1-D discrete Fourier transform. Here each submatrix $P_{\theta_{k}}$ is a sparse matrix that computes the discrete approximations of projections of $f$ at angle $\theta_{k}$. Since $F$ is orthogonal, we can write $\mathbf{b}=F^{*} \hat{\mathbf{f}}$, which allows us to simplify the above system to

$$
\mathbf{b}=A \mathbf{f},
$$

Note that (4) is essentially the tomographic formulation of SAR [23, 26]. A window function matrix, $S$, is commonly used to reduce the sidelobe artifacts caused by the incompleteness of the Fourier data, yielding

$$
\mathbf{b}_{S}=F^{*} S \hat{\mathbf{f}}=A \mathbf{f} .
$$

\subsection{Numerical Linear Operators for SAR}

Due to simplicity, back projection is often used to form SAR images (see [20]). Unfortunately, back projection often yields streaking and sidelobe artifacts in the reconstructed image, and does not adequately reduce the effects of noise. As an alternative, let us discuss how one might attempt to solve (4) directly ${ }^{4}$ Note that finding a solution via gradient descent requires an efficient evaluation of both the forward operator and its adjoint (see e.g. [30]). Although $A$ is itself sparse in each row, most SAR data sets include hundreds of pulses sent over an aperture of very small degree, yielding thousands of azimuth angles. Thus constructing $A$ is prohibitively time consuming $5^{5} \mathrm{~A}$ faster alternative is to implement the nonuniform fast Fourier transform (NUFFT), which efficiently

\footnotetext{
${ }^{4}$ Although not practical in real applications, it is instructive to consider the direct approach in the development of our $\ell_{1}$ regularization algorithm.

${ }^{5}$ We note that our investigation does not focus on direct solvers of $\sqrt[4]{4}$, and there may indeed be faster algorithms available.
} 

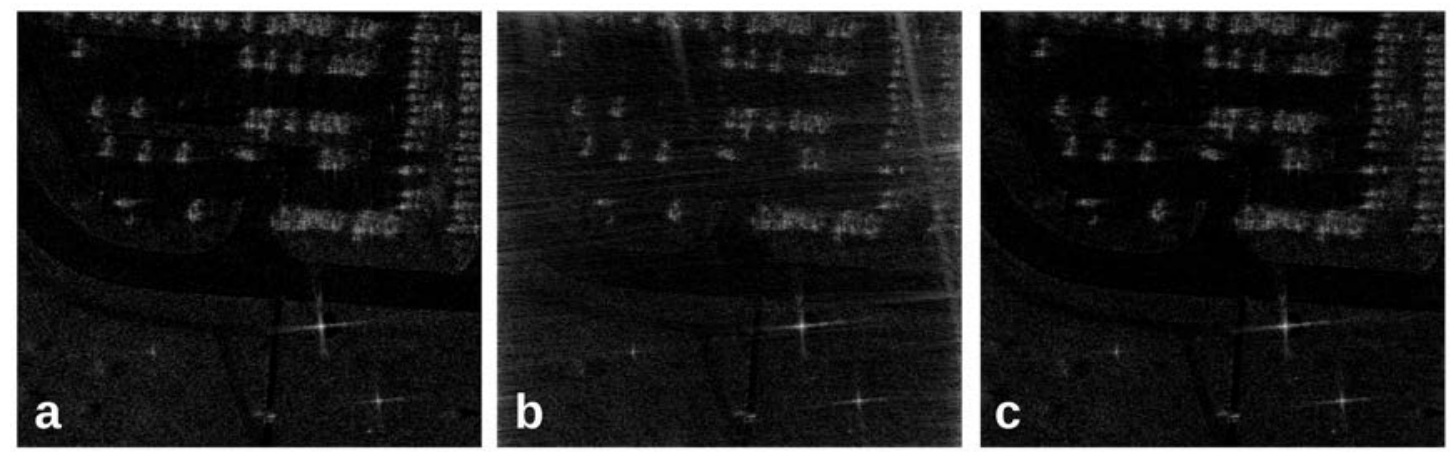

Figure 1: Reconstruction of the GOTCHA data set [2] over a $10^{\circ}$ aperture using the (a) back projection; (b) least squares; and (c) least squares with a quadratic penalty term (Tikhonov regularization).

approximates $F A$, leading to real time image formation. In short, the NUFFT implements discrete convolution to regrid the nonuniform $k$-space locations onto a uniform grid so that the FFT can be applied. Numerous studies have been devoted to the NUFFT, (see e.g. 8, 21, 4), and in our work we have used the algorithms written in [17.

Figure 1 compares three standard image reconstruction algorithms applied to the GOTCHA data set [2] over a $10^{\circ}$ aperture. Observe that the straightforward approach of solving (4) via least squares in Figure 1(b) produces more noise and error than the back projection method (Figure 1 (a)), even though the former is more consistent with the model itself. This is particularly evident in the upper right quadrant of the image. Regularization via a quadratic penalty term helps to mitigate some of these effects (Figure 11(c)).

\subsection{Regularization for $S A R$}

Solving ill-posed linear systems such as (4) typically requires some form of regularization to handle issues of noise, incompleteness of data, and in this case, error in the forward model resulting in sidelobes and specular noise. The regularization is commonly carried out by

$$
J(\mathbf{f})=\frac{\mu}{2}\|F A \mathbf{f}-\hat{\mathbf{f}}\|_{2}^{2}+H(\mathbf{f})
$$

where $H(\mathbf{f})$ is typically some norm or semi-norm acting as the regularization term that penalizes unfavorable solutions. In general, $H$ is only required to be convex so that $J(\mathbf{f})$ is also convex. Hence iterative methods are guaranteed to terminate near a global minimum. Figure 1(c) displays 

difference between all adjacent pixels.

Recently it has become popular to encourage sparsity of $\mathbf{f}$ in some appropriate "smoothness" domain by setting $H(\mathbf{f})=\|T \mathbf{f}\|_{1}$, where $T$ a linear mapping under which smoothly varying functions are assumed to be sparse. For example, the total variation (TV) semi-norm, $\|D \mathbf{f}\|_{1}$, which encourages sparsity in the edges of the image, is used in a variety of applications. Often in SAR, the reflective function $\mathbf{f}$ is just assumed to contain a sparse number isotropic point scatterers, so that an appropriate regularization term is simply $\|\mathbf{f}\|_{1}$.

\subsubsection{Phase Estimation}

With complex valued scenes, conventional TV regularization would enforce smoothness on both the real and imaginary parts of the scene. However, smoothness in SAR images is characterized by the magnitude of the scatterers, and, due to a variety of factors, such as the discretization of an underlying continuous problem, modeling errors, and noise in the data, little structure can be found in the phase of the image pixels. Therefore it is much more appropriate to characterize the smoothness of SAR images in terms of the image magnitude, $|\mathbf{f}|$. Unfortunately, because of its nonlinearity, the operator $|\cdot|$ can not be implemented seamlessly in optimization algorithms. To overcome this issue, we follow the approach recently introduced in [27, 12], which involves first estimating the phase angles of $\mathbf{f}$ and then building a diagonal unitary matrix $\Theta$, so that $\Theta^{*} \mathbf{f} \approx|\mathbf{f}|$. Here $\Theta_{j, j}=\operatorname{angle}\left(\tilde{\mathbf{f}}_{j}\right)$ and $\tilde{\mathbf{f}}$ is some initial approximate solution. This has been called phase estimation and leads to the objective function

$$
J(\mathbf{f})=\frac{\mu}{2}\|F A \mathbf{f}-\hat{\mathbf{f}}\|_{2}^{2}+\left\|D \Theta^{*} \mathbf{f}\right\|_{1} .
$$

Another alternative uses the absolute value explicitly in the formulation by modifying the objective functional, [10]. However, the non-linearity of this operation adds significant complexity to the optimization procedure.

\subsection{Techniques for fast implementation}

Because a more complete model would require solving the nonlinear inverse problem to Maxwell's equations, [14, the Fourier assumption used to construct (4) and (6) is practical in that it allows 
for computational efficiency ${ }^{6}$ Still, numerical implementation of the regularized model $(6)$ is not straightforward and algorithms must be designed for its robust and accurate calculation. As will be shown in our numerical results, the so called alternating direction method of multipliers (ADMM), and specifically the one designed in [24, provides an efficient algorithm for computing [6]. In particular, we solve

$$
\min _{f, g}\left\{\frac{\mu}{2}\|F A f-\hat{f}\|_{2}^{2}+\frac{\beta}{2}\left\|D \Theta^{*} f-g\right\|_{2}^{2}+\|g\|_{1}-\left\langle\sigma, D \Theta^{*} f-g\right\rangle\right\}
$$

A detailed description of the algorithm used for solving (7) is given in Appendix A A few important aspects are discussed below.

The variable splitting given by the introduction of $g$ in (7) allows for a fast alternating minimization scheme over $f$ and $g$, [24, 19]. Compared with standard $\ell_{2}$ minimization solvers, here the image is "softened" or "smoothed" with each shrinkage step over $g$, while iteratively moving to a smaller $\ell_{2}$ error data fit given by $f$. The Lagrange multiplier, $\sigma$, and the second $\ell_{2}$ term in the functional are used to enforce the equality constraint $D \Theta^{*} f=g$.

When the forward operator, $F A$, is far from orthogonal, a large number of iterations are needed to realize convergence of the $\ell_{2}$ data term. In our case, due to the orthogonality of $F$ and the sparsity of the rows in $A$, convergence of the algorithm can be realized after relatively few iterations, and in our experiments, we have found the algorithm generally converges between 20 and 30 iterations. The parameter $\beta$ is used to ensure that the variable splitting formulation encourages $D \Theta^{*} f=g$. However 115 this constraint is inevitably enforced by the last term after the Lagrangian multiplier is updated several times, so that the parameter $\beta$ fairly robust. In our experiments we use $\beta=32$ unless otherwise specified. The balance between the data fitting and the regularization is determined by $\mu$. To this end, a significant distinction in our formulation is the absence of a Lagrangian multiplier term to enforce the data fit (in other work referred to as "adding back the noise", [19]). This prevents 120 the overfitting of the data, which is especially important given the errors discussed previously of the SAR model.

We summarize the discussion by highlighting the major differences in our $\ell_{1}$ optimization for SAR compared with traditional $\ell_{1}$ ADMM algorithms applied to other problems, such as tomo-

\footnotetext{
${ }^{6}$ Other models (see e.g [15]) are well developed but have not yet been efficiently implemented for SAR.
} 


\begin{tabular}{c|c|c|c} 
& NUFFT & Back projection & $\ell_{1}(20$ iterations $)$ \\
\hline $\mathrm{N}=352$ & $.052 \mathrm{~s}$ & $5.01 \mathrm{~s}$ & $8.52 \mathrm{~s}$ \\
$\mathrm{~N}=821$ & $.078 \mathrm{~s}$ & $12.02 \mathrm{~s}$ & $10.01 \mathrm{~s}$ \\
$\mathrm{~N}=1407$ & $.142 \mathrm{~s}$ & $20.66 \mathrm{~s}$ & $14.44 \mathrm{~s}$ \\
\hline
\end{tabular}

Table 1: Run times for each reconstruction approach as a function of $N$, the number of pulses or angles.

graphic imaging:

1. The data fit parameter $\mu$ should be relatively small to account for inaccuracies in the model.

2. There is no "adding back the noise" or use of a Lagrangian multiplier for the data fitting.

3. As will be demonstrated in our numerical experiments, relatively few iterations are needed to realize convergence, e.g. 20 or 30.

Figure 2 shows results of implementing (7) from a $4^{\circ}$ aperture of the GOTCHA data set 2 .

130 with an increased number of pulses. 
a
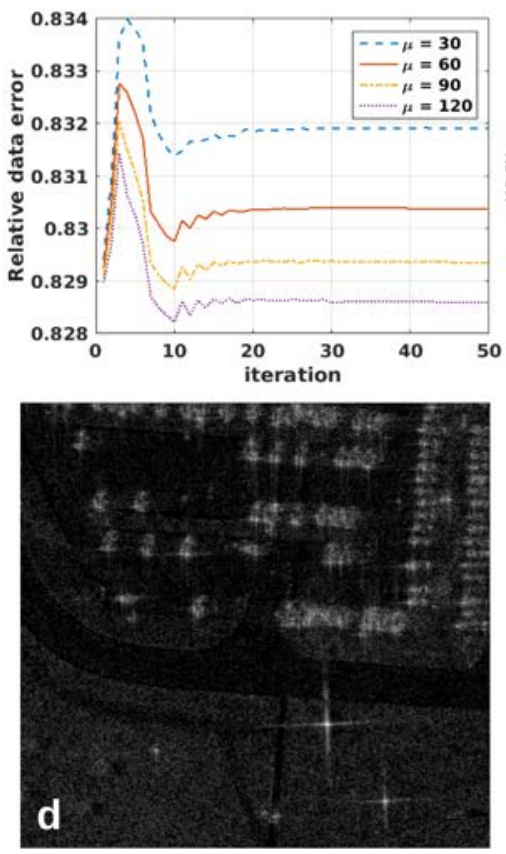

b
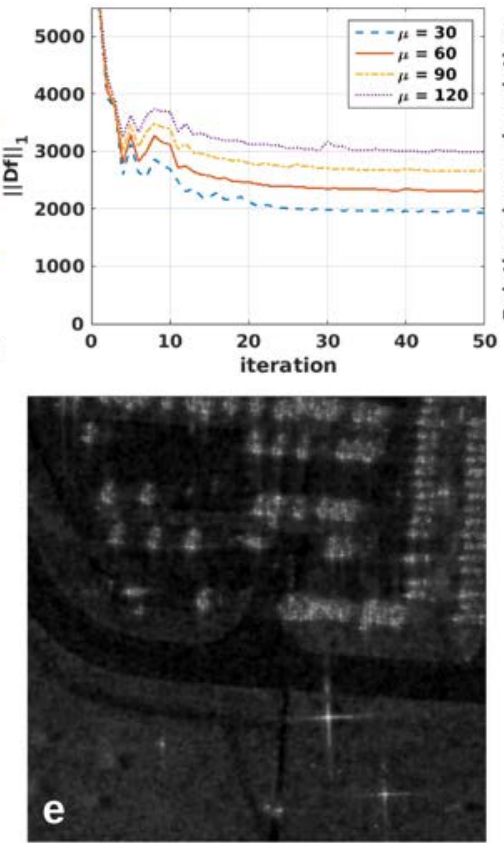

C
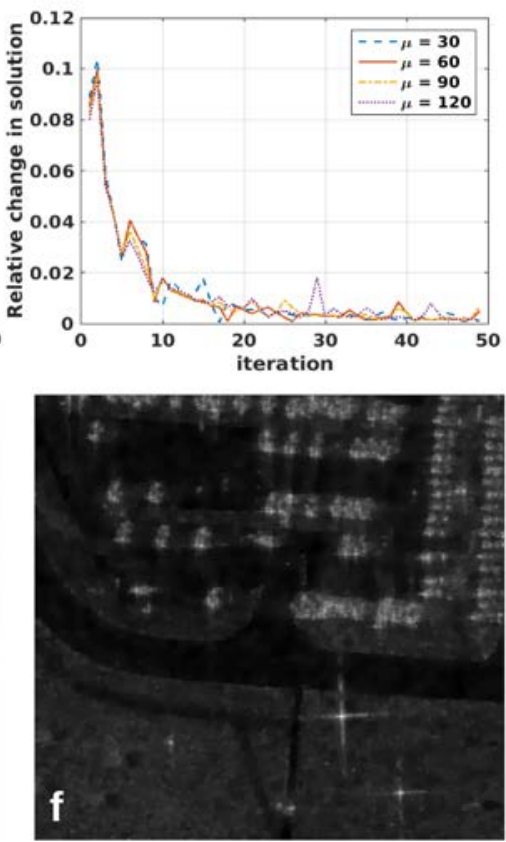

Figure 2: Reconstruction of a GOTCHA data set over a $4^{\circ}$ data set using 7 with $\beta=32$. (a) $\ell_{2}$ and (b) and $\ell_{1}$ terms for different choices of $\mu$ as a function of iterations; (c) Convergence of the solution as a function of iterations; (d) Back projection reconstruction of (4); Solution using (7) after (e) 10 and (f) 50 iterations. 


\section{Robust Composite Image Formation}

In the general derivation of the SAR imaging problem, it is assumed that scene contains only

\subsection{Review of Composite Image Formation}

Composite image formation, introduced in 25, 11, to alleviate some of the issues observed in Figure 3. creates a final composite image from the angularly dependent responses to yield higher signal response. The method is performed by first dividing the full available wide-angle aperture into $K$ sub-aperture bins. It is reasonable to assume isotropic scattering within the angular extent of the sub-apertures. The image is then recovered in each bin from the corresponding viewing angle to generate a sequence of $K$ images. Once the sub-aperture images $\mathbf{f}^{k}$ for $k \in\{1,2, \ldots, K\}$ have
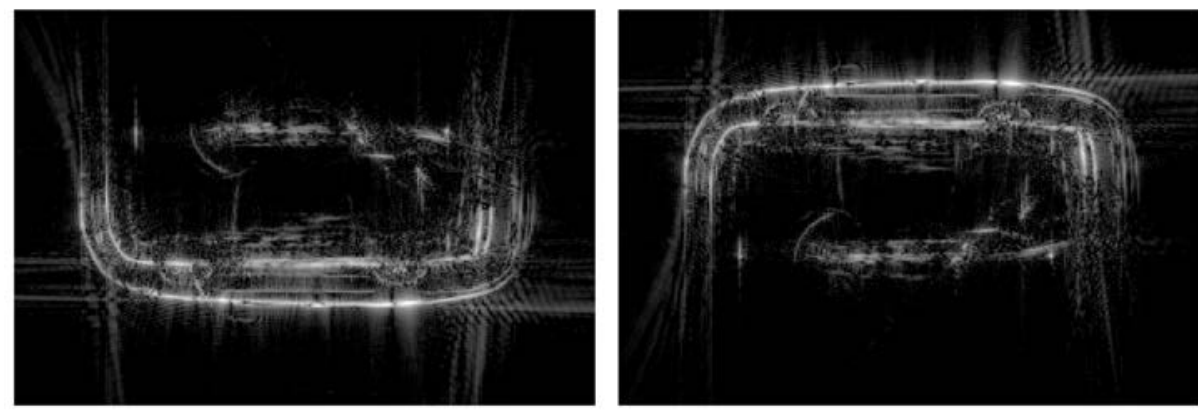

Figure 3: Back projection reconstruction of a CV dome data set of a Toyota Camry, [1] performed by splitting the full azimuth into two separate data sets, $\left[0,180^{\circ}\right],\left[180^{\circ}, 360^{\circ}\right]$. 
been formed, the $(i, j)$ pixel of the composite image is given by

$$
\mathbf{f}_{i, j}=\arg \max _{k} \mathbf{f}_{i, j}^{k}
$$

where here it is implied that only the magnitude of the complex valued images is considered.

Originally each of the $K$ images was constructed using back projection or matched filtering, [25]. As an extension of the wide-angle reconstruction discussion, one might expect better results by regularizing each individual image construction. However, in previous investigations, perhaps due to prohibitive computational cost, the $\ell_{1}$ regularization was only implemented as a post-processing procedure via a deconvolution model with the point spread function, [25, 11]. By contrast, our algorithms in Appendix A and Appendix B yield a cost efficient reconstruction that includes $\ell_{1}$ regularization in each iteration. Figure 4 compares direct and composite imaging for back projection and $\ell_{1}$ sparsity regularization. Observe the increased sharpness and reduced speckle for both the composite imaging and $\ell_{1}$ regularization reconstructions.

Relatively little discussion has focused on the computational efficiency of composite imaging for $\mathrm{SAR}$, and indeed, since its introduction, $\ell_{1}$ regularization and NUFFT algorithms have undergone significant improvements in efficiency and robustness, [19, 24, 17, 21]. Table 2 provides run times

for wide angle and composite imaging using state of the art imaging algorithms. In both cases, the NUFFT is most efficient. Using $\ell_{1}$ regularization with NUFFT operators has a run time comparable to back projection.

\section{Using Joint Sparsity in Composite Imaging}

We are now able to discuss the main new contribution of this investigation. First, let us rewrite the $\ell_{1}$ regularized composite imaging solution as

$$
\mathbf{f}^{k *}=\arg \min _{\mathbf{f}^{k}}\left\{\left\|F_{k} \mathbf{f}^{k}-\hat{\mathbf{f}}^{k}\right\|_{2}^{2}+\mu\left\|\mathbf{f}^{k}\right\|_{1}\right\}
$$

where $F_{k}$ are the discrete forward operators for each small sub-aperture, which again can be computed with the NUFFT. Note that the phase estimation terms are not necessary in (8) since sparsity in the image domain is independent of the phase. Observe that this sequence of $K$ optimizations is 

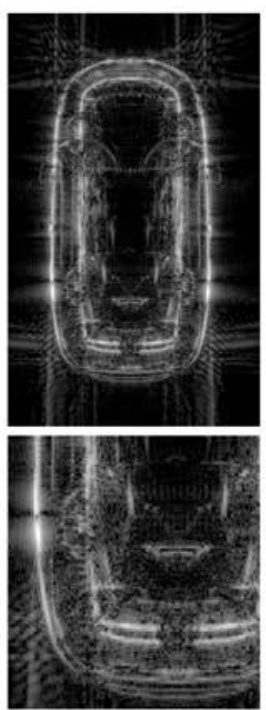

(a)
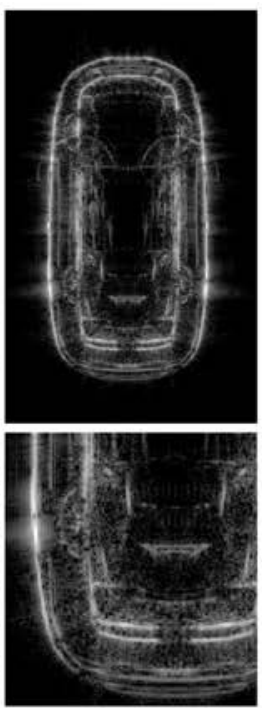

(b)
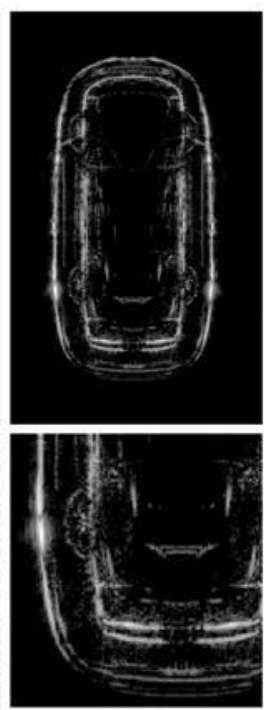

(c)
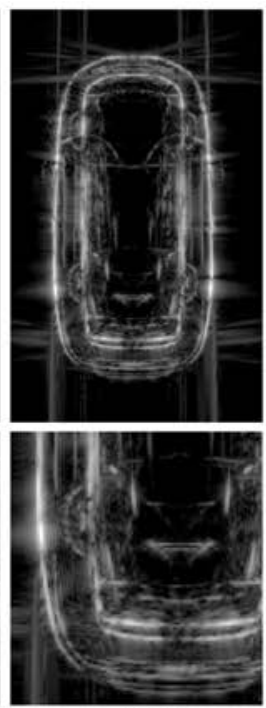

(d)
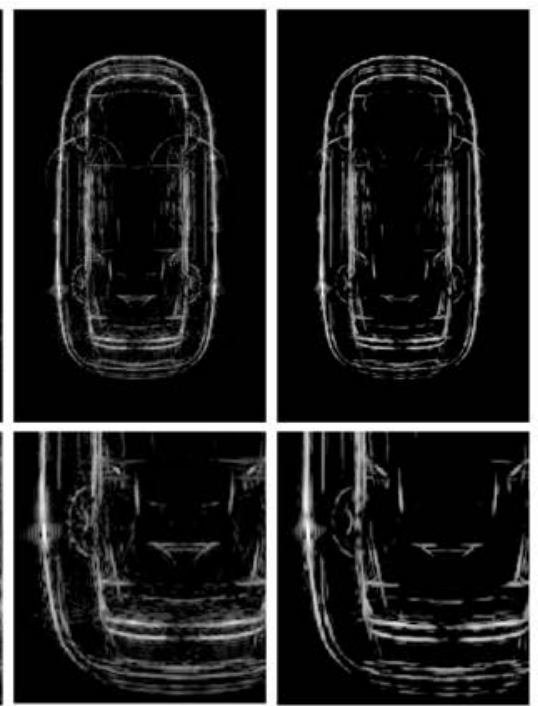

(e)

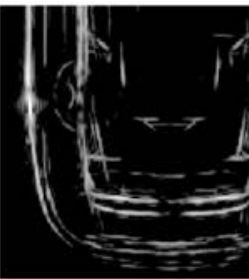

(f)

Figure 4: Direct imaging with full azimuth using (a) back projection, (b) NUFFT, and (c) $\ell_{1}$ regularization. Composite imaging using (d) back projection and (e) $\ell_{1}$ regularization. (f) Reconstruction from joint sparsity model, 11. In all cases we chose $\beta=32$ with $60 \leq \mu \leq 120$.

equivalent to the single optimization problem given by

$$
\left(\mathbf{f}^{1 *}, \mathbf{f}^{2 *}, \ldots, \mathbf{f}^{K *}\right)=\underset{\left(\mathbf{f}^{1}, \mathbf{f}^{2}, \ldots, \mathbf{f}^{K}\right)}{\operatorname{argmin}}\left\{\sum_{k=1}^{K}\left(\frac{\mu}{2}\left\|F_{k} \mathbf{f}^{k}-\hat{\mathbf{f}}^{k}\right\|_{2}^{2}+\left\|\mathbf{f}^{k}\right\|_{1}\right)\right\}
$$

\subsection{Sequential joint sparsity}

With the joint sparsity formulation, we can implement additional terms to make use of the overlapping information that relates the sequence of images. For neighboring apertures, the recovered images, $\mathbf{f}^{k *}$ and $\mathbf{f}^{(k+1) *}$ as determined by $[8$, should contain similar edge information. However, both will still contain some unwanted speckle and sidelobe artifacts. To further reduce these unwanted artifacts while retaining the important edge information, we suggest using a joint sparsity approach, [18]. In a nutshell, joint sparsity suggests that a set (or subset) of images have similar support in their sparsity domain. Such information can be exploited through additional constraints leading to a more robust recovery for each individual image, and the subsequent composite 
reconstruction. One option is to include a TV-like norm of the form

$$
\left\|\Theta_{k+1}^{*} \mathbf{f}^{k+1}-\Theta_{k}^{*} \mathbf{f}^{k}\right\|_{1}
$$

for each $k$. As in (6), $\Theta_{k}$ are diagonal matrices with the estimated phase at each pixel of $\mathbf{f}^{k}$. Placing (10) into 99 yields

$$
\left(\mathbf{f}^{1 *}, \mathbf{f}^{2 *}, \ldots, \mathbf{f}^{K *}\right)=\underset{\left(\mathbf{f}^{1}, \mathbf{f}^{2}, \ldots, \mathbf{f}^{K}\right)}{\operatorname{argmin}}\left\{\sum_{k=1}^{K}\left(\frac{\mu}{2}\left\|F_{k} \mathbf{f}^{k}-\hat{\mathbf{f}}^{k}\right\|_{2}^{2}+\left\|\mathbf{f}^{k}\right\|_{1}\right)+\gamma \sum_{k=1}^{K-1}\left\|\Theta_{k+1}^{*} \mathbf{f}^{k+1}-\Theta_{k}^{*} \mathbf{f}^{k}\right\|_{1}\right\} .
$$

Intuitively, (11) should significantly reduce speckle and unwanted sidelobe artifacts. In particular, in neighboring images such artifacts which are often random and/or highly angularly sensitive will not coincide, whereas the main edge features will exhibit significant overlap. With a full $360^{\circ}$ azimuth, $\mathbf{f}^{1}$ and $\mathbf{f}^{K}$ should also share overlapping information and can be included as an additional difference term. An algorithm for computing (11) is provided in Appendix B.

We note that the joint sparsity approach we have introduced here is modified in several ways from the prototype (see e.g. [18), which usually assumes that the overlapping support is between all images and globally enforced. It is also commonly enforced through tight constraint equalities rather than additional penalty terms. Indeed, in other related SAR work, 29, 31, the images reconstructed from different elevation angles are constrained to having the same magnitude at each pixel location. In our investigation we are assuming the same elevation of the $K$ images, however. Moreover, based on the discussion surrounding Figure 3 it is evident that not all of the $K$ images share the same support. Indeed, there is "local" as opposed to "global" joint sparsity, and hence we enforce a local penalty term. Finally, in our formulation we are able to exploit the overlapping support for each individual reconstruction, which is more effective than when it is used only for post-processing the combined results.7 We refer to our approach as sequential joint sparsity.

Figure 4 compares the various approaches discussed in this investigation, and includes both direct and composite imaging using $\ell_{1}$, back projection, and our new (sequential) joint sparsity approach. We again used a $4 \mathrm{Ghz}$ bandwidth with a central frequency of $10 \mathrm{Ghz}$. Each of the composite images retains the structure of true edges while also reducing the effects of specular

\footnotetext{
${ }^{7}$ We note that the joint space aspect approach in 35] exploits a related idea. However, the method described there does not use phase estimation and the regularization term is non-linear.
} 

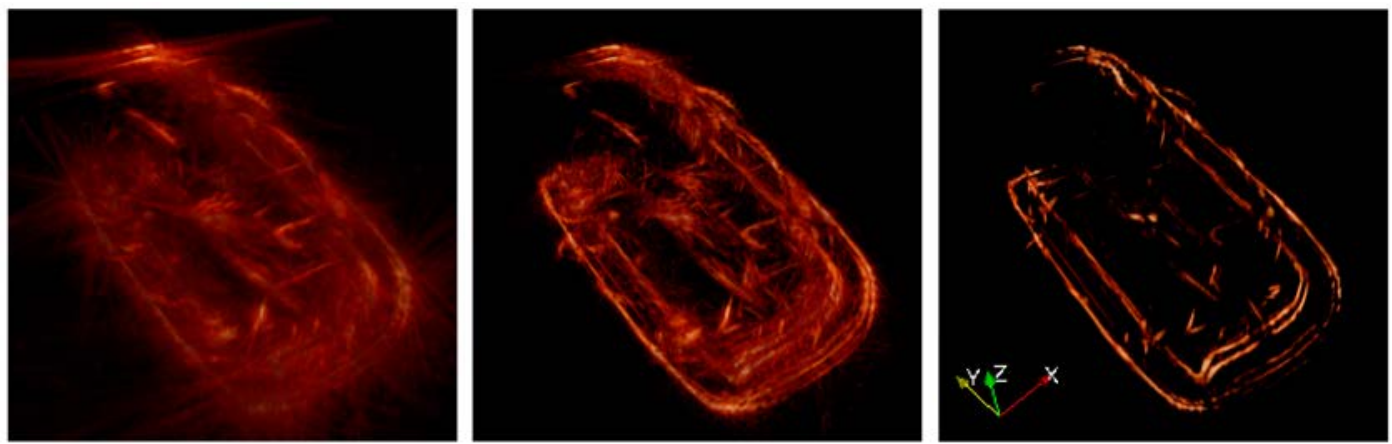

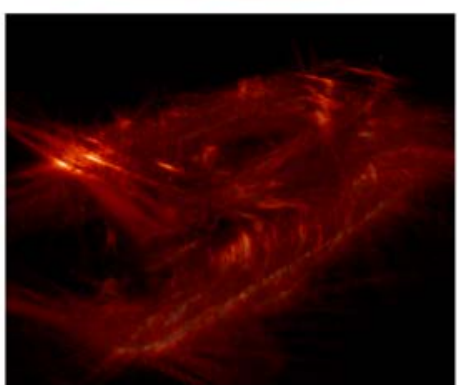

(a)

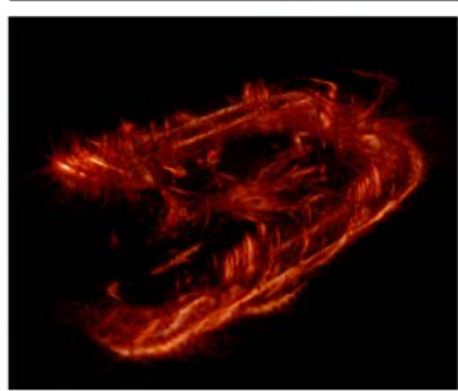

(b)

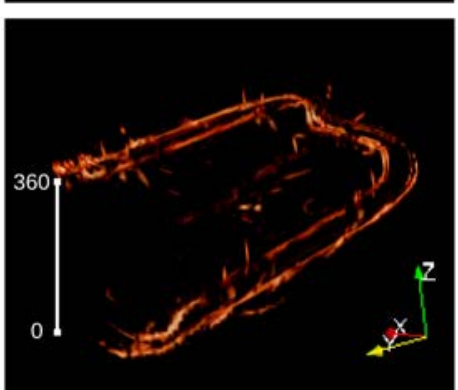

(c)

Figure 5: 3-D comparison the of composite image techniques using (a) back projection; (b) $\ell_{1}$ regularization; and (c) our joint sparsity approach. The visuals were generated by viewing the sequence of reconstructed 2-D images from the varying apertures as a 3-D stack using the tomviz software [3. The $z$-axis represents the azimuth angle used to reconstruct each sub-aperture image. 


\begin{tabular}{c|c|c|c|c} 
& NUFFT & Back projection & $\ell_{1}$ & Joint sparsity \\
\hline Direct Imaging & $21.3 \mathrm{~s}$ & $84.6 \mathrm{~s}$ & $41.4 \mathrm{~s}$ & $\mathrm{n} / \mathrm{a}$ \\
Composite & $41.9 \mathrm{~s}$ & $139.0 \mathrm{~s}$ & $142.4 \mathrm{~s}$ & $127.3 \mathrm{~s}$ \\
\hline
\end{tabular}

Table 2: Run times for each reconstruction approach from the full $360^{\circ}$ azimuth. We used 20 iterations for the (direct) $\ell_{1}$ regularization approach 7 and 20 iteration the composite approach. In all cases $\beta=32$ and $60 \leq \mu \leq 120$.

noise. The image resulting from the joint sparsity approach shown in Figure 4(e) also seems to have almost no speckle or sidelobe artifacts. Some of the image features appear to be faded, however no attempt to fine tune the parameters has been made.

In order to better understand the accuracy of each of the composite images, Figure 5 shows 3 -D visuals using the composite approach with back projection, $\ell_{1}$ regularization, and (sequential) joint sparsity. In particular, we consider the sequence of reconstructed 2-D images over the varying azimuths stacked together to form a 3-D matrix, subsequently yielding a 3-D image. This allows us to better view the varying reconstructions as functions of the azimuth range. Running along the $z$-axis, indicated in the right column of Figure 5, is the change is the azimuth range. In the $x y$ planes are each $f^{k}$ image, for $k=1, \ldots, K$, where in this example $K=45$ and each image was formed using a $10^{\circ}$ azimuth with a $1^{\circ}$ overlap in the azimuth of the neighboring images. These images agree with those in Figure 4, showing that the standard $\ell_{1}$ regularization (shown in Figure 5(b)) reduces much of the sidelobe and speckle present in the back projection (shown in Figure 5(a)). Most notably however, the sequential joint sparsity approach (shown in Figure 5(c)) removes nearly all of the unwanted artifacts while still capturing the main features of the Camry. We note that it is easy to modify the two new parameters introduced here, degree of sub-aperture and degree of overlap. This will be investigated in future work. We have not found our results to be sensitive for our particular data sets in this regard.

The run time for the joint sparsity approach is provided in the last column of Table 2 . We used 20 iterations for the standard $\ell_{1}$ approach and 10 iterations for the composite $\ell_{1}$ approach in $(8)$.

While the run times may vary depending on the number of iterations per time step, our numerical experiments strongly indicate that the image quality obtained using the joint sparsity approach is generally improved. Moreover, it may be possible to develop more efficient parallel algorithms. 

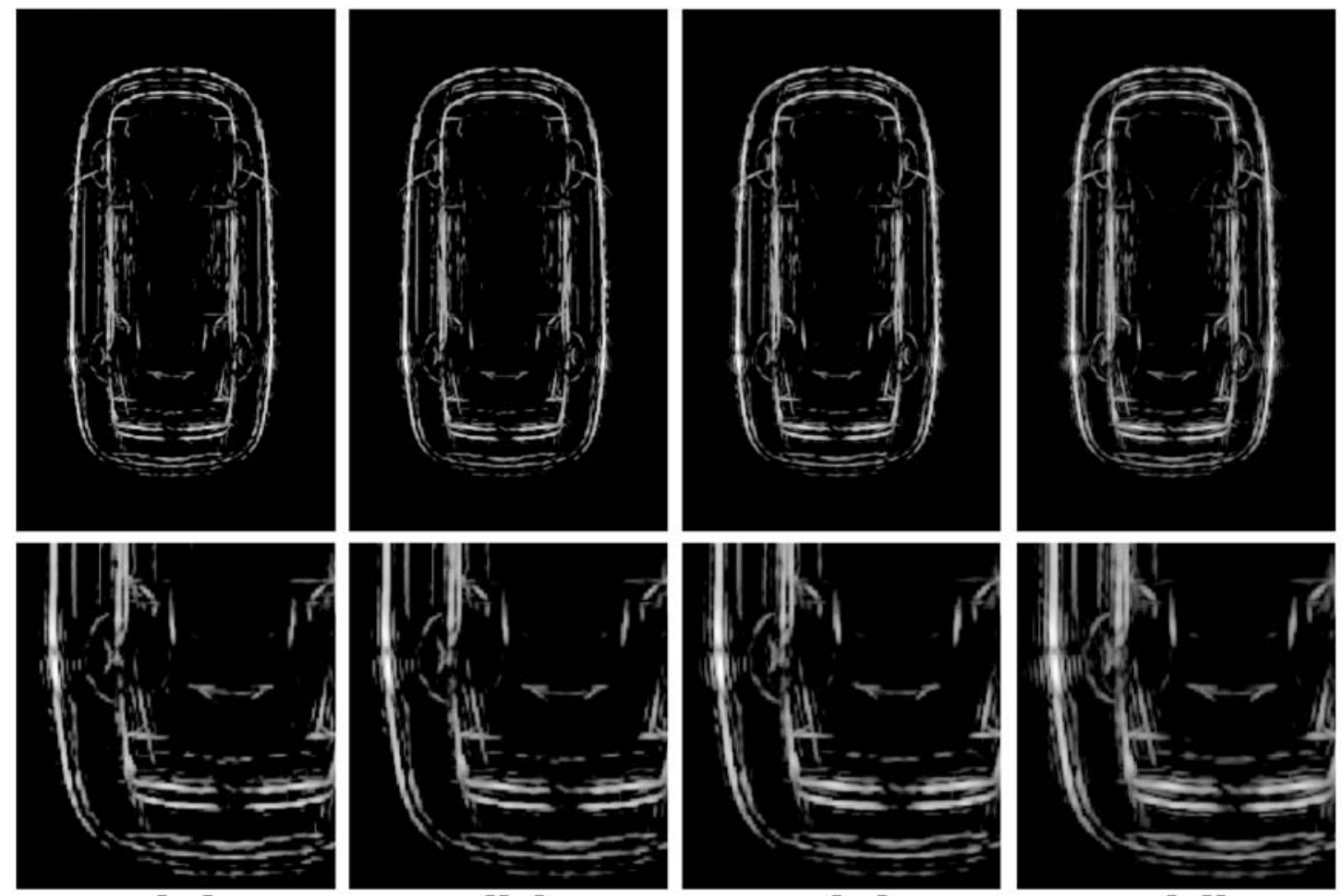

(a)

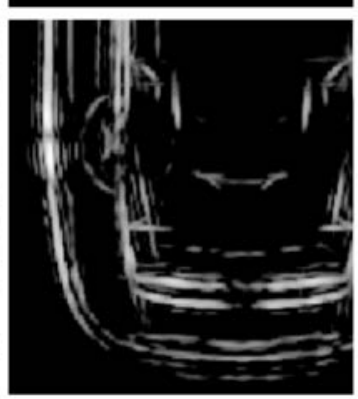

(c)

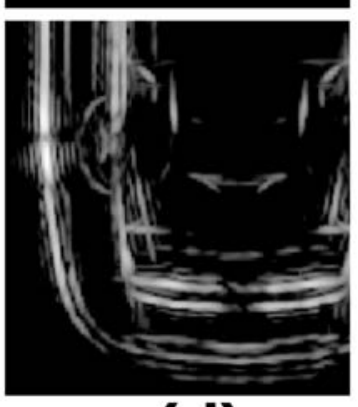

(d)

Figure 6: Joint sparsity approach 12 using PA of order 1 (a), 1.5 (b), 2 (c), and 2.5 (d). 


\subsection{Generalizing the Joint Sparsity Approach via Higher and Fractional Order Finite Differences}

The joint sparsity approach introduced an additional TV-like term between the sequence of sub-aperture images. While TV, which can be considered as a first order finite difference of an image, is effective in many applications, other less restrictive higher order finite difference models that promote a more refined edge map can also be implemented. In particular we note that while TV essentially annihilates constants, that is zeroth order polynomials, higher order finite differences annihilate higher order polynomials. Such operators, termed polynomial annihilation (PA) in [5], and higher order total variation (HOTV) in [13, can be used directly in the joint sparsity model yielding

$\left(\mathbf{f}^{1 *}, \mathbf{f}^{2 *}, \ldots, \mathbf{f}^{K *}\right)=\underset{\left(\mathbf{f}^{1}, \mathbf{f}^{2}, \ldots, \mathbf{f}^{K}\right)}{\operatorname{argmin}}\left\{\sum_{k=1}^{K}\left(\frac{\mu}{2}\left\|F_{k} \mathbf{f}^{k}-\hat{\mathbf{f}}^{k}\right\|_{2}^{2}+\left\|\mathbf{f}^{k}\right\|_{1}\right)+\gamma \sum_{k=1}^{K-\ell}\left\|\sum_{m=0}^{\ell}(-1)^{m+\ell}\left(\begin{array}{c}\ell \\ m\end{array}\right) \Theta_{k+m}^{*} \mathbf{f}^{k+m}\right\|_{1}\right\}$.

Here $\ell$ is the order of the PA transform with $\ell=1$ reducing to TV, as in (11).

This approach can also be generalized further by implementing fractional order finite differences (FD), that is non-integer values $\ell$, as in [16]. Since the fractional derivatives can be calculated via FFT in Fourier space, there is little additional computational cost. The fractional order FD scheme can be briefly described as follows:

Consider the integer order $\ell \mathrm{FD}$ as a convolution with the vector $\mathbf{v}$ given by

$$
\mathbf{v}_{m}:=\left\{\begin{array}{ll}
(-1)^{m+\ell}\left(\begin{array}{c}
\ell \\
m
\end{array}\right) & \text { if } 0 \leq m \leq \ell \\
0 & \text { if } \ell<m<N
\end{array} .\right.
$$

Then the convolution can be calculated as a product in the Fourier domain, with the Fourier transform of $\mathbf{v}$ directly given as

$$
\hat{\mathbf{v}}_{n}=\sum_{m=0}^{\ell}(-1)^{m+\ell}\left(\begin{array}{c}
\ell \\
m
\end{array}\right) e^{-i \frac{2 \pi}{N} m n}=\left(e^{-i \frac{2 \pi}{N} n}-1\right)^{\ell}, \quad \text { for } \quad n=0,1, \ldots, N-1
$$

From here, fractional order derivatives can be computed via an inverse Fourier transform of each

225 vector component $\hat{\mathbf{f}}_{n} \hat{\mathbf{v}}_{n}$ for any positive non-integer value $\ell$ using standard FFT algorithm. With the appropriate construction of the operator $D$, a straightforward modification of Algorithms 1 and 2 can be used to solve 12]. A more detailed description and MATLAB code is available at [33]. 
Figure 6 compares the joint sparsity model $(12)$ using orders $1,1.5,2$, and 2.5 for the CV dome set of a Toyota Camry [1]. Notice that the fading of the artifacts becomes less evident as the order of FD is increased, but sidelobe and speckle artifacts increase due to the oscillatory nature of high order FD. Yet these artifacts are still not as prevalent as when the joint sparsity model is not used, suggesting that some parameter tuning based on prior information can improve the results. For this example it appears that $1.5 \leq \ell \leq 2.5$ recovers the best results.

In order to compare wide angle and composite imaging, Figure 7 shows the reconstruction of the GOTCHA data set [2] over the full $360^{\circ}$ azimuth ${ }^{8}$ In contrast to the CV Dome Camry, this scene suggests that the first $\ell_{1}$ term in 12 be replaced with TV-like $\ell_{1}$ norm as in (6). It is immediately evident that the composite imaging approach yields better resolution than wide angle SAR. Further, when using composite imaging, employing TV (middle row, right column) does not significantly improve the results obtained by using back projection (middle row, left column). However, the joint sparsity approach (bottom row) appears to both recover important features and reduce artifacts. Notice, for example, the point-like scatterers which appear at the bottom right of the scene. These point-like scatterers appear to be better localized in the joint sparsity image, while there are still some ringing artifacts in the standard composite images. In our experiments we have observed that noise-like artifacts in the composite images are typically reduced when using joint sparsity, which is also consistent with the results pertaining to the CV Dome Camry data set.

While our examples consistently demonstrate improved results using the composite approach with sequential joint sparsity, quantifiable comparisons are difficult since there are no exact solutions corresponding to our data sets. Moreover, simulating the data from a known image using a quadrature formulation of 2 would yield an artificially close match to the Fourier model, and as a consequence would not demonstrate the full usefulness of the composite joint sparsity approach, which was introduced to allow for deviation from that model. A more suitable approach for simulating SAR images can be found in 32 . Figure 8 (a) shows the ideal scattering response of five flat plates placed on the ground, each three meters long and two meters high. The data are generated using the MATLAB code provided in 32 on 512 equally spaced frequencies over a 2 Ghz bandwidth centered at $\omega_{0}=9.6 \mathrm{Ghz}$ and 0 elevation angle. We compare the back projection, composite imaging with $\ell_{1}$ regularization, and the sequential joint sparsity technique in Figures 8 (b-d),(b1-d1)

\footnotetext{
${ }^{8}$ The first of the eight available data sets was used.
} 


\section{Direct Imaging}
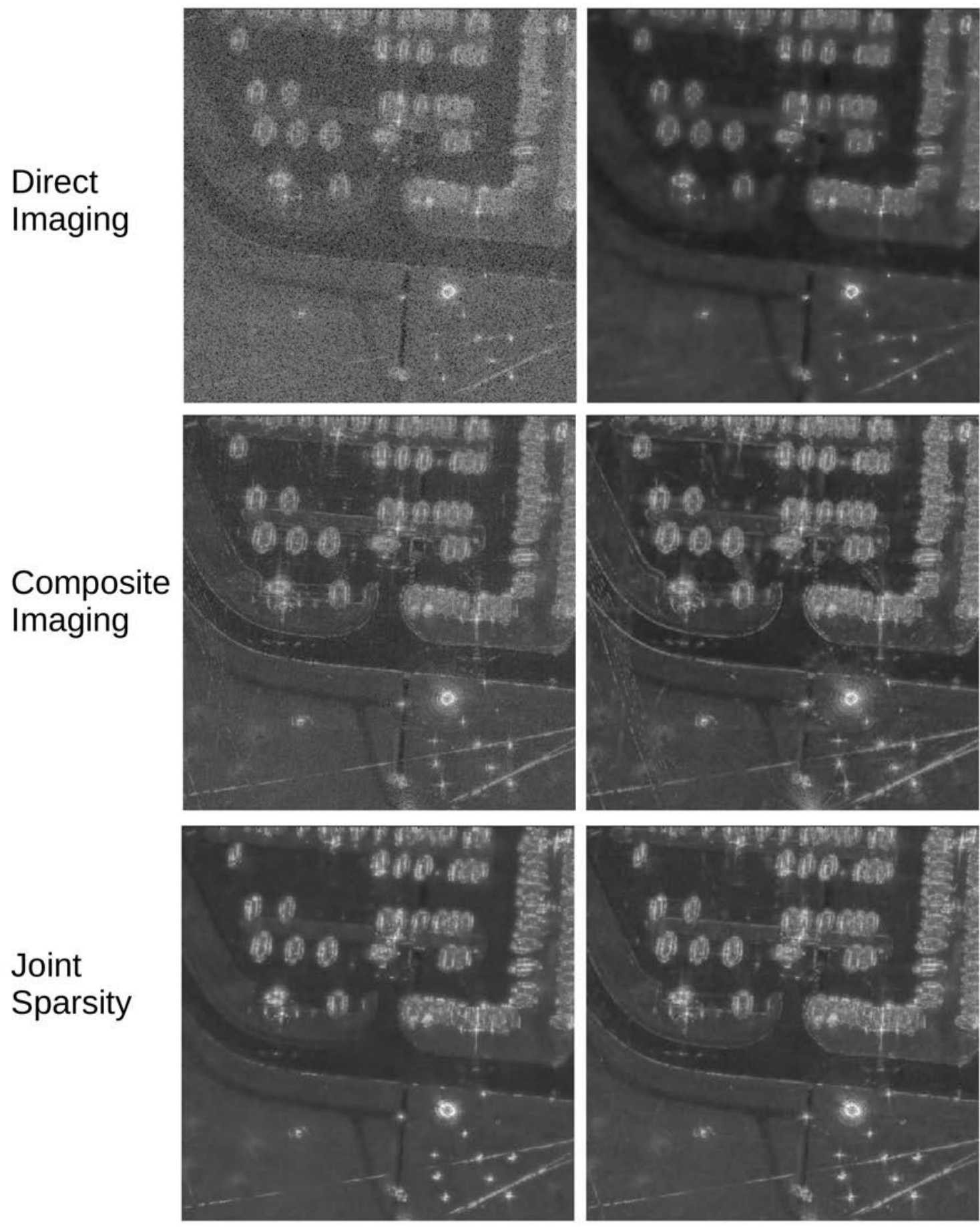

Figure 7: Reconstruction of the GOTCHA data set 2 from a full $360^{\circ}$ azimuth. The direct and composite imaging results were formed using back projection (left) and TV regularization (right). The sequential joint sparsity results were formed using 12 with orders of 1 (left) and 3 (right). 

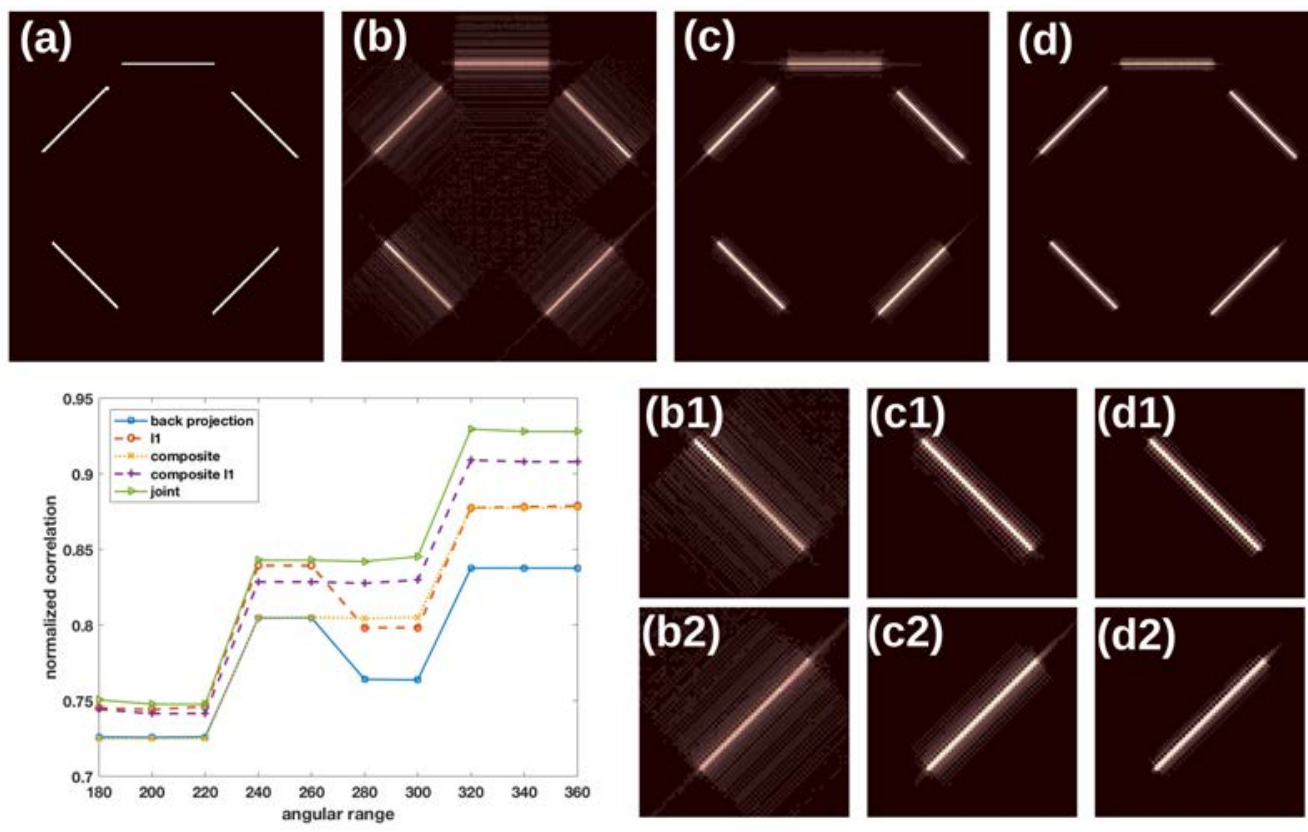

Figure 8: (a) Ideal scattering response. Reconstructions from the full $360^{\circ}$ azimuth using (b) back projection, (c) composite imaging with $\ell_{1}$ regularization, and (d) our new joint-sparsity model in (11). For closer inspection the corresponding reconstructed walls in the lower half of the scene are magnified in (b1)-(d1) and (b2)-(d2).

and (b2-d2). The correlation, given by

$$
\frac{<\mathbf{f}, \tilde{\mathbf{f}}>}{\|\tilde{\mathbf{f}} \mid\| \tilde{\mathbf{f}} \|},
$$

where $\mathbf{f}$ is the ideal scatter response and $\tilde{\mathbf{f}}$ is a given reconstruction, is shown for increasing angular range. It is evident that the sequential joint sparsity composite approach achieves the highest correlation, which is consistent with what we have already observed in previous examples. Indeed, Figure 8 does not likely demonstrate how well our method compares under less ideal circumstances. Due to the simplicity of the underlying image, HOTV is not needed here.

\section{Summary}

In this investigation we designed $\ell_{1}$ regularization algorithms using phase estimation for SAR imaging. Compared with other traditional regularized inverse problems, we found that less emphasis on the data fitting $\left(\ell_{2}\right.$ term) produces a better SAR model, leading to fewer required iterations. Moreover, by employing the robust NUFFT, the computational run times are comparable 
to traditional back projection. Our numerical experiments also indicates that the $\ell_{1}$ regularization algorithm consistently yields higher image quality.

We then applied these techniques to composite imaging construction, and furthermore designed a sequential joint sparsity model by regularizing the results between the sequence of images. This provides the most notable improvement, with significant speckle and sidelobe artifact reduction. The joint sparsity model was also generalized for greater flexibility and robustness in the approach, and our numerical experiments confirm the advantage in using HOTV, although more work is needed to determine which $\ell_{1}$ regularizer should be employed. Our new method is numerically efficient and is easily implemented using the ADMM.

\section{Appendix A. Algorithm 1}

The algorithms described in these sections are available in [33]. The difficulty in solving [6] comes from the non-differentiability of the $\ell_{1}$ norm. A commonly used clever approach for solving similar problems is to reformulate the minimization as in (7), which is sometimes called the augmented Lagrangian functional. We first provide the alternating minimization algorithm for solving (7), where we rewrite the objective function as

$$
J(\mathbf{f}, \mathbf{g}, \sigma ; \mu, \beta)=\left\{\frac{\mu}{2}\|F A \mathbf{f}-\hat{\mathbf{f}}\|_{2}^{2}+\frac{\beta}{2}\left\|D \Theta^{*} \mathbf{f}-\mathbf{g}\right\|_{2}^{2}+\|\mathbf{g}\|_{1}-\left\langle\sigma, D \Theta^{*} \mathbf{f}-\mathbf{g}\right\rangle\right\} .
$$

270

The following pseudo algorithm is carried out for minimizing $J$ over $\mathbf{f}$ and $\mathbf{g}$ :

Efficient minimization of $\mathbf{f}$ and $\mathbf{g}$ is critical to Algorithm 1. Exact numerical minimization over $\mathbf{f}$ can be quite costly, and therefore an aggressive one-step steepest decent approach is implemented to give a reliable estimate [24]. Note that the feasibility of this approach is due to the differentiability of all terms involving $\mathbf{f}$. Indeed, the gradient of $J$ with respect to $\mathbf{f}$ is given by

$$
\nabla_{\mathbf{f}} J(\mathbf{f}, \mathbf{g}, \sigma ; \mu, \beta)=\mu(F A)^{*}(F A \mathbf{f}-\hat{\mathbf{f}})+\beta\left(D \Theta^{*}\right)^{*}\left(D \Theta^{*} \mathbf{f}-\mathbf{g}\right)-\left(D \Theta^{*}\right)^{*} \sigma .
$$




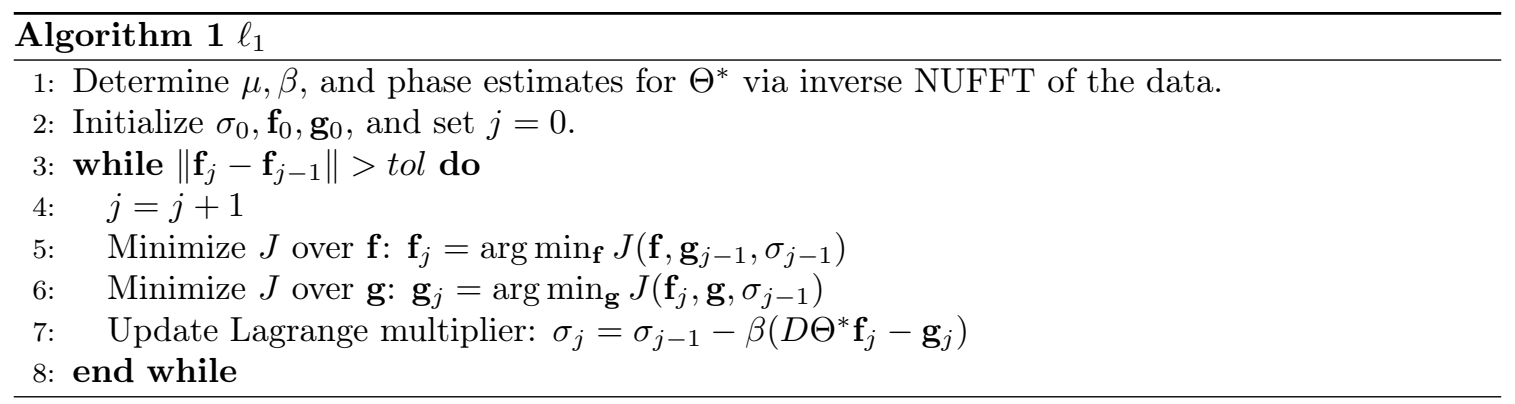

The step length used is the so called BB-like step length [9].

Conveniently, minimization over $\mathbf{g}$ is provided by an exact formula (see [24]) that can be calculated efficiently as

$$
\mathbf{g}_{j}=\max \left(\left|D \Theta^{*} \mathbf{f}_{j}-\frac{\sigma_{j-1}}{\beta}\right|-\frac{1}{\beta}, 0\right) * \operatorname{sgn}\left(D \Theta^{*} \mathbf{f}_{j}-\frac{\sigma_{j-1}}{\beta}\right)
$$

The update over the Lagrange multiplier is done in order to enforce the constraint $D \Theta^{*} f=g$, so that we are approximately minimizing the original functional in 6 .

\section{Appendix B. Algorithm 2}

We now address a similar problem of approximating the solution to (11) using an augmented Lagrangian function. Although this formulation appears to be more complicated, with a few simple changes we can apply Algorithm 1. We first let

$$
F=\left(\begin{array}{cccc}
F_{1} & 0 & \ldots & 0 \\
0 & F_{2} & \ldots & 0 \\
\vdots & & \ddots & \vdots \\
0 & & \ldots & F_{K}
\end{array}\right), \quad \mathbf{f}=\left(\begin{array}{c}
\mathbf{f}^{1} \\
\mathbf{f}^{2} \\
\vdots \\
\mathbf{f}^{K}
\end{array}\right), \quad \text { and } \quad \hat{\mathbf{f}}=\left(\begin{array}{c}
\hat{\mathbf{f}}^{1} \\
\hat{\mathbf{f}}^{2} \\
\vdots \\
\hat{\mathbf{f}}^{K}
\end{array}\right)
$$

Let $T$ be the operator that maps $\mathbf{f}$ into all of the $\ell_{1}$ sparsity terms appearing in (11), which can 
be written as

$$
T \mathbf{f}=\left(\begin{array}{c}
\mathbf{f} \\
\gamma *\left(\Theta_{2}^{*} \mathbf{f}_{2}-\Theta_{1}^{*} \mathbf{f}_{1}\right) \\
\gamma *\left(\Theta_{3}^{*} \mathbf{f}_{3}-\Theta_{2}^{*} \mathbf{f}_{2}\right) \\
\vdots \\
\gamma *\left(\Theta_{K}^{*} \mathbf{f}_{K}-\Theta_{K-1}^{*} \mathbf{f}_{K-1}\right)
\end{array}\right) .
$$

Then indeed $T$ is a linear operator which can be calculated via matrix multiplication. However, it is more practical to define $T$ through a series of operations, such as MATLAB's function handle approach. With these variables, (11) now equivalently reads

$$
\min _{\mathbf{f}}\left\{\frac{\mu}{2}\|F \mathbf{f}-\hat{\mathbf{f}}\|_{2}^{2}+\|T \mathbf{f}\|_{1}\right\} .
$$

275 with $T$ from B.1. Algorithm 1 then immediately applies.

\section{References}

[1] Civilian vehicle data dome overview. https://www.sdms.afrl.af.mil/index.php? collection=cv_dome. Accessed: 2016-19-08.

[2] GOTCHA volumetric SAR data set overview. https://www.sdms.afrl.af.mil/index.php? collection=gotcha. Accessed: 2016-19-08.

[3] tomviz software for tomographic visualization of 3D scientific data. http://www.tomviz.org/ Accessed: 2016-06-09.

[4] F. Andersson, R. Moses, and F. Natterer. Fast Fourier methods for synthetic aperture radar imaging. IEEE Transactions on Aerospace and Electronic Systems, 48(1):215-229, 2012.

[5] R. Archibald, A. Gelb, and R. B. Platte. Image reconstruction from undersampled Fourier data using the polynomial annihilation transform. Journal of Scientific Computing, 67(2):432-452, 2016.

[6] R. Archibald, A. Gelb, and J. Yoon. Polynomial fitting for edge detection in irregularly sampled signals and images. SIAM Journal on Numerical Analysis, 43(1):259-279, 2005. 
[7] F. Argenti, A. Lapini, T. Bianchi, and L. Alparone. A tutorial on speckle reduction in synthetic aperture radar images. IEEE Geoscience and Remote Sensing Magazine, 1(3):6-35, 2013.

[8] A. Averbuch, R. Coifman, D. Donoho, M. Elad, and M. Israeli. Fast and accurate polar Fourier transform. Applied and Computational Harmonic Analysis, 21(2):145 - 167, 2006.

[9] J. Barzilai and J. M. Borwein. Two-point step size gradient methods. IMA Journal of Numerical Analysis, 8(1):141-148, 1988.

[10] M. Cetin and W. C. Karl. Feature-enhanced synthetic aperture radar image formation based on nonquadratic regularization. IEEE Transactions on Image Processing, 10(4):623-631, 2001.

[11] M. Cetin and R. L. Moses. SAR imaging from partial-aperture data with frequency-band omissions. In Defense and Security, pages 32-43. International Society for Optics and Photonics, 2005.

[12] M. Cetin, O. Onhon, and S. Samadi. Handling phase in sparse reconstruction for SAR: Imaging, autofocusing, and moving targets. In Synthetic Aperture Radar, 2012. EUSAR. 9th European Conference on, pages 207-210, 2012.

[13] T. Chan, A. Marquina, and P. Mulet. High-order total variation-based image restoration. SIAM Journal on Scientific Computing, 22(2):503-516, 2000.

[14] M. Cheney and B. Borden. Fundamentals of Radar Imaging. Society for Industrial and Applied Mathematics, 2009.

[15] D. Colton and R. Kress. Inverse Acoustic and Electromagnetic Scattering Theory. Springer, 1992.

[16] F. Dong and Y. Chen. A fractional-order derivative based variational framework for image denoising. Inverse Problems \& Imaging, 10(1), 2016.

[17] J. Fessler and B. Sutton. Nonuniform fast Fourier transforms using min-max interpolation. IEEE Transactions on Signal Processing, 51(2):560-574, 2003.

[18] M. Fornasier and H. Rauhut. Recovery algorithms for vector-valued data with joint sparsity constraints. SIAM Journal on Numerical Analysis, 46(2):577-613, 2008. 
[19] T. Goldstein and S. Osher. The split Bregman method for 11-regularized problems. SIAM Journal on Imaging Sciences, 2(2):323-343, 2009.

[20] L. A. Gorham and L. J. Moore. SAR image formation toolbox for matlab. In SPIE Defense, Security, and Sensing, pages 769906-769906, 2010.

[21] L. Greengard and J.-Y. Lee. Accelerating the nonuniform fast Fourier transform. SIAM Review, 46(3):443-454, 2004.

[22] C. V. Jakowatz, D. E. Wahl, P. H. Eichel, D. C. Ghiglia, and P. A. Thompson. Range Resolving Techniques, pages 1-31. Springer US, Boston, MA, 1996.

[23] C. V. Jakowatz, D. E. Wahl, P. H. Eichel, D. C. Ghiglia, and P. A. Thompson. A Tomographic Foundation for Spotlight-Mode Sar Imaging, pages 33-103. Springer US, Boston, MA, 1996.

[24] C. Li, W. Yin, H. Jiang, and Y. Zhang. An efficient augmented Lagrangian method with applications to total variation minimization. Comput. Optim. Appl., 56(3):507-530, 2013.

[25] R. L. Moses, L. C. Potter, and M. Cetin. Wide-angle SAR imaging. In Defense and Security, pages 164-175. International Society for Optics and Photonics, 2004.

[26] F. Natterer, M. Cheney, and B. Borden. Resolution for radar and x-ray tomography. Inverse Problems, 19(6):S55, 2003.

[27] K.-Y. Ni and S. Rao. SAR moving target imaging using sparse and low-rank decomposition. In SPIE Defense+ Security, pages 90771D-90771D. International Society for Optics and Photonics, 2014.

[28] C. Paulson. Utilizing Glint Phenomenology to Perform Classification of Civilian Vehicles Using Synthetic Aperture Radar. PhD thesis, University of Florida, 2013.

[29] L. C. Potter, E. Ertin, J. T. Parker, and M. Cetin. Sparsity and compressed sensing in radar imaging. Proceedings of the IEEE, 98(6):1006-1020, 2010.

[30] M. J. D. Powell. Restart procedures for the conjugate gradient method. Mathematical Programming, 12(1):241-254, 1977. 
[31] N. Ramakrishnan, E. Ertin, and R. L. Moses. Enhancement of coupled multichannel images using sparsity constraints. Trans. Img. Proc., 19(8):2115-2126, 2010.

[32] B. D. Rigling. Raider tracer: a matlab-based electromagnetic scattering simulator. SPIE Radar Modeling and Measurement, 6568, 2007.

[33] T. Sanders. Matlab imaging algorithms: Image reconstruction, restoration, and alignment,

口 with a focus in tomography. http://www.toby-sanders.com/software, https://doi.org/ 10.13140/RG.2.2.33492.60801. Accessed: 2016-19-08.

[34] T. Sanders, A. Gelb, R. B. Platte, I. Arslan, and K. Landskron. Recovering fine details from 350 under-resolved electron tomography data using higher order total variation 11 regularization. Ultramicroscopy, 174:97-105, 2017.

[35] I. Stojanovic, M. Cetin, and W. C. Karl. Joint space aspect reconstruction of wide-angle sar exploiting sparsity. In SPIE Defense and Security Symposium, pages 697005-697005. International Society for Optics and Photonics, 2008. 